\title{
Non-Invasive Detection of Anastomotic Leakage Following Esophageal and Pancreatic Surgery by Urinary Analysis
}

\author{
Victor D. Plat ${ }^{a}$ Nora van Gaal ${ }^{b}$ James A. Covington ${ }^{c}$ Matthew Neal ${ }^{d}$ \\ Tim G.J. de Meij ${ }^{\mathrm{e}}$ Donald L. van der Peet ${ }^{\mathrm{a}} \quad$ Babs Zonderhuis $^{\mathrm{a}}$ Geert Kazemier $^{\mathrm{a}}$ \\ Nanne K.H. de Boer ${ }^{b}$ Freek Daams ${ }^{a}$ \\ ${ }^{a}$ Department of Gastrointestinal surgery, VU University Medical Center, Amsterdam, The Netherlands; \\ ${ }^{b}$ Department of Gastroenterology and Hepatology, VU University Medical Center, Amsterdam, The Netherlands; \\ 'School of Engineering, University of Warwick, Coventry, UK; ' Department of Statistics, University of Warwick, \\ Coventry, UK; ${ }^{e}$ Department of Pediatric Gastroenterology and Hepatology, VU University Medical Center, \\ Amsterdam, The Netherlands
}

\section{Keywords}

Pancreatic surgery · Esophageal surgery $\cdot$ Anastomotic leakage $\cdot$ Volatile organic compounds · Field asymmetric ion mobility spectrometry

\section{Abstract \\ Background: Esophagectomy or pancreaticoduodenecto- my is the standard surgical approach for patients with tu- mors of the esophagus or pancreatic head. Postoperative mortality is strongly correlated with the occurrence of anas- tomotic leakage (AL). Delay in diagnosis leads to delay in treatment, which ratifies the need for development of novel and accurate non-invasive diagnostic tests for detection of AL. Urinary volatile organic compounds (VOCs) reflect the metabolic status of an individual, which is associated with a systemic immunological response. The aim of this study was to determine the diagnostic accuracy of urinary VOCs to de- tect AL after esophagectomy or pancreaticoduodenectomy. Methods: In the present study, urinary VOCs of 63 patients after esophagectomy $(n=31)$ or pancreaticoduodenectomy}

( $n=32)$ were analyzed by means of field asymmetric ion mobility spectrometry. AL was defined according to international study groups. Results: AL was observed in 15 patients (24\%). Urinary VOCs of patients with AL after pancreaticoduodenectomy could be distinguished from uncomplicated controls, area under the curve 0.85 (95\% Cl 0.76-0.93), sensitivity $76 \%$, and specificity $77 \%$. However, this was not observed following esophagectomy, area under the curve 0.51 (95\% Cl 0.37-0.65). Conclusion: In our study population AL following pancreaticoduodenectomy could be discriminated from uncomplicated controls by means of urinary VOC analysis, NTC03203434.

(c) 2018 The Author(s)

Published by S. Karger AG, Basel

\section{Introduction}

Anastomotic leakage (AL) is the most dreaded and frequent complication after intestinal surgery, leading to significantly increased morbidity and 30-day mortality [1]. According to the Dutch Institute for Clinical Audit-

\begin{tabular}{ll}
\hline KARGER & $\begin{array}{l}\text { @ } 2018 \text { The Author(s) } \\
\text { Published by S. Karger AG, Basel }\end{array}$ \\
E-Mail karger@karger.com & $\begin{array}{l}\text { This article is licensed under the Creative Commons Attribution- } \\
\text { NonCommercial-NoDerivatives 4.0 International License (CC BY- } \\
\text { NC-ND) (http://www.karger.com/Services/OpenAccessLicense). } \\
\text { Usage and distribution for commercial purposes as well as any dis- } \\
\text { tribution of modified material requires written permission. }\end{array}$
\end{tabular}

Victor D. Plat, BSc

Department of Gastrointestinal Surgery

VU University Medical Center

De Boelelaan 1117, ZH 7F020, NL-1081 HV Amsterdam (The Netherlands)

E-Mail V.plat@vumc.nl 
ing, leakage occurs in $12.8 \%$ of pancreaticoduodenectomies and 20\% of esophagectomies carried out in the Netherlands [2].

In current practice, AL is diagnosed based on clinical, biochemical, and radiological findings. In the early postoperative phase, clinical parameters have been shown to be indecisive, requiring additional examination [3]. Primary biochemical predictor is the acute phase $\mathrm{C}$-reactive protein (CRP). However, serum CRP lacks sensitivity and specificity for major infectious complications [4]. In addition, increased levels of amylase in drain fluid have been shown to be predictive for esophageal [5] and pancreatic AL [6]. Nevertheless, early drain removal is favorable for the postoperative recovery, preventing secondary infection and theoretically pancreatitis [7]. This emphasizes the need for the development of novel, non-invasive biomarkers, with high accuracy for predicting and diagnosing $\mathrm{AL}$ during the first postoperative days.

Since 2000 BC, ancient physicians have been diagnosing patients based on their own olfactory system [8]. Most gases, either odorous or non-odorous, are composed of volatile organic compounds (VOCs). These compounds have several origins: firstly, environmental or exogenous; secondly, local symptoms from the primary affected disease site in diseased patients which is often inflammation or cancer; and thirdly, the systemic (immunological) response [8]. Assessment of VOCs in breath, feces, and urine has led to the identification of a variety of disease-specific smell prints in, for example, pulmonology, oncology, and gastroenterology [9-14].

The aim of this study was to determine the diagnostic accuracy of analysis of urinary VOCs to detect AL noninvasively after major gastrointestinal surgery.

\section{Methods}

\section{Study Design and Participants}

This study was conducted in accordance with the World Medical Association Declaration of Helsinki. The local institutional ethical review board of the VU medical center approved the study protocol (2014.543). This project was designed as a single center prospective observational cohort study between March 2015 and April 2016. Eligible participants were diagnosed with (suspected) malignancies of the esophagus, pancreas, distal bile duct, ampulla of Vater, or duodenum and were scheduled for surgery (i.e., esophagectomy or pancreaticoduodenectomy). Patients were aged 1890 years and had an American Society of Anesthesiologists physical status of 3 or lower. All participants were capable of understanding the study information, and signed written informed consent. Patient, surgery, and pathology data were prospectively registered.

\section{Procedure}

Patients were recruited from the department of surgery of the VU University medical center (Amsterdam, The Netherlands). They were admitted to the hospital 1 day prior to surgery and received standard preoperative treatment according to local protocol. Patients diagnosed with esophageal cancer underwent the following procedures: Ivor Lewis, McKeown, or transhiatal esophagectomy. For pancreatic cancer, a pancreaticoduodenectomy was performed according to Whipple or with preservation of the gastric pylorus (pylorus-preserving pancreaticoduodenectomy $[\mathrm{PPPD}])$. All procedures were performed under standardized antibiotic prophylaxis (cefuroxime/metronidazole). In case of a pancreaticoduodenectomy, a prophylactic drain was placed at the pancreaticojejunostomy. Postoperatively, all patients were treated according to local protocol, which consisted of admittance to the intensive care unit, medium care, or general ward. Drains were removed when drain amylase content was within normal range on or after postoperative day 3 . Patients did not receive additional antibiotics during the first 3 postoperative days.

\section{Endpoints}

The primary endpoint of this study concerned clinical postoperative AL. Leakage following esophageal surgery was defined by the Esophagectomy Complications Consensus Group as a full thickness defect involving the esophageal anastomosis. Severity was graded based on required treatment. Grade 1 was a local defect only requiring medical treatment with dietary modification. Grade 2 required interventional but not surgical therapy, and grade $3 \mathrm{AL}$ was surgically treated [15]. Leakage of the pancreaticojejunostomy was defined as any measurable volume of drain fluid on or after postoperative day 3 , with amylase content more than 3 times the upper normal serum volume. The International Study Group on Pancreatic Fistula graded leakage according to clinical impact. A biochemical leak (formerly grade A) has by definition no clinical impact and was therefore not listed as clinical AL. Grade B requires a change in therapeutic management of the expected postoperative pathway. Grade $\mathrm{C}$ consists of AL-related organ failure or clinical instability such that a reoperation was needed [16]. Furthermore, other postoperative complications were prospectively registered. For this study, a 30-day morbidity was determined and all complications were detected using local protocol, consisting of daily physical and routine biochemical examinations. When clinical signs were indicative for AL, radiologic or endoscopic imaging was requested to objectify.

\section{Sample Collection}

Urinary samples were collected in $4.5 \mathrm{~mL}$ containers (Cryopure ${ }^{\circledR}$ ) on postoperative day 1,2 , and 3 . All samples were obtained from urinary catheters while maintaining a sterile system. Subsequently, samples were frozen at $-80^{\circ} \mathrm{C}$ within $2 \mathrm{~h}$ of collection for simultaneous batch analysis.

\section{Field Asymmetric Ion Mobility Spectrometry of Urinary Gas}

Urinary gas analysis was performed using a commercial field asymmetric ion mobility spectrometry (FAIMS) unit (Lonestar, Owlstone, UK). FAIMS utilizes high electric fields to separate different ionized chemicals based on their movement (called mobility) in this high electric field. The unit was fitted with an ATLAS sampling system (Owlstone UK), which heats the urine sample to $38^{\circ} \mathrm{C}$ and controls the flow of clean air over the top of the sample and into the Lonestar instrument. The flow rate of the carrier gas 


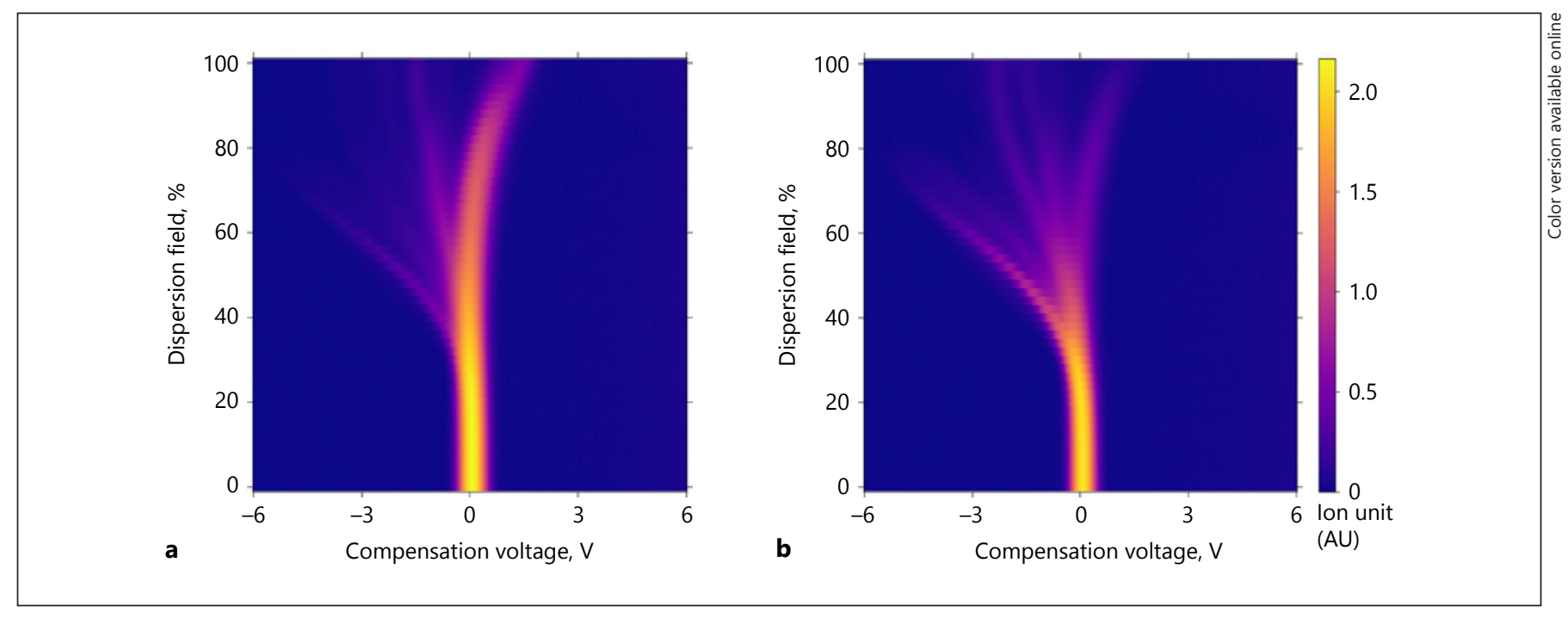

Fig. 1. Urinary VOC data generated using FAIMS from an AL patient (a) and an uncomplicated patient (b) following pancreaticoduodenectomy. FAIMS measures positive and negative ion counts at a range of compensation voltages ( -6 to $+6 \mathrm{~V}$ in 512 steps) and electric field magnitudes (called the dispersion field, $0-100 \%$ in 51

(dry clean air) over the sample was $500 \mathrm{~mL} / \mathrm{min}$, with a total flow into the Lonestar of $2 \mathrm{~L} / \mathrm{min}$. The inlet of the Lonestar was heated to $100^{\circ} \mathrm{C}$ to ensure that no condensation occurred. Inside the unit, headspace VOCs are ionized using a NI-63 radiation source and passed between 2 parallel plates. Onto these plates a high-electric field is applied, which attracts, repels, or does not affect the ions. Ions that touch one of the plates lose their charge and are not detected. A compensation voltage is added to one of the plates to counteract this movement. By scanning through a range of compensation voltages (+6 to $-6 \mathrm{~V}$ in 512 steps), a wide range of different mobilities were measured. Furthermore, the magnitude of the electric field is scanned through a range of levels (called the dispersion field), which was increased in 51 steps. Three scans of the compensation voltage and dispersion field strength were performed for each sample. By measuring positive and negative ions, a total of 156,672 data points were collected per sample. Further details of this analysis have been described previously [17].

\section{Analysis}

Samples were stored in the freezer for a maximum of 12 months. Levels of VOCs start to reduce after 9 months of storage [18]. Samples were removed from the freezer to defrost at room temperature for a maximum of $2 \mathrm{~h}$. Before analysis, the $4.5 \mathrm{~mL}$ of sample was aliquoted into a $22 \mathrm{~mL}$ glass vial, placed in the Lonestar, and heated to $38^{\circ} \mathrm{C}$. All samples were measured in triplicate. Before and after each urine sample, a sample of clean tap water was run 3 times to ensure that the baseline response was returned. Glass vials were sanitized at the department of microbiology after each sample.

\section{Statistical Analysis}

Demographic and disease specific data will be tabulated. Urinary VOCs of patients with AL were compared to control patients who had an uncomplicated postoperative course for both types of steps) to generate 52,224 data points per scan. Three scans are performed per sample. This figure shows the negative ion counts for the final scan. Distinct plumes in the image correlate to the presence of distinct VOCs in the sample. VOCs, volatile organic compounds.

surgery. Data of the 3 separate days were combined for both groups before comparison. IBM SPSS statistics (version 23) was used for standard statistical analysis. All continuous variables are expressed as median and range; percentages were calculated for dichotomous variables. When appropriate, baseline characteristics were compared with an independent sample $t$ test or Fisher's exact test. The FAIMS dataset was analyzed using our existing pipeline developed for previous studies (13). In brief, first a denoising step was applied to the FAIMS data, followed by a data compression algorithm to reduce the dimensionality of the data. Ten-fold cross-validation was then used to assess classifier performance. Figure 1 shows urinary VOC data of a patient in the AL group and an uncomplicated patient following pancreaticoduodenectomy. The displayed samples are closest to the mean for their group (AL or uncomplicated) and therefore representative of the average result.

Dimensionality reduction was undertaken using a 2D wavelet transform (specifically Daubechies D4 wavelets) and any resulting variables with zero variance were excluded. A 10-fold cross-validation was then performed, using $90 \%$ of the data as a training set, and the remaining $10 \%$ as a test set. Within each fold, 64 informative features were identified using a Wilcoxon rank-sum test on the training set. These 64 features were then used to train a random forest classifier and predict the probability of AL for the samples in the test set. A separate initial cross-validation was used for model selection, comparing sparse logistic regression, radial SVM, linear SVM, and stochastic gradient boosting. All models except linear SVM showed statistically significant predictive ability (at a 95\% significance level) of AL after pancreaticoduodenectomy and of these, random forest was found to produce the best classification results using area under the receiver operator curve (AUROC) as a metric. 
Table 1. Summary of demographic and clinical characteristics of patients undergoing esophageal surgery

\begin{tabular}{|c|c|c|}
\hline Characteristics & $\begin{array}{l}\mathrm{AL} \\
(n=9)\end{array}$ & $\begin{array}{l}\text { Controls } \\
(n=22)\end{array}$ \\
\hline \multicolumn{3}{|l|}{ Gender } \\
\hline Male & $9(100)$ & $16(73)$ \\
\hline Female & $0(0)$ & $6(27)$ \\
\hline Age, years & $70(40-81)$ & $61(46-79)$ \\
\hline Missing urine samples & $3(11)$ & $1(2)$ \\
\hline \multicolumn{3}{|l|}{ Location of the anastomosis } \\
\hline Cervical & $2(22)$ & $8(36)$ \\
\hline Thoracic & $7(78)$ & $14(64)$ \\
\hline \multicolumn{3}{|l|}{ Type of carcinoma } \\
\hline Adenocarcinoma & $5(56)$ & $16(73)$ \\
\hline Squamous cell carcinoma & $1(11)$ & $5(23)$ \\
\hline Other & $3(33)$ & $1(5)$ \\
\hline \multicolumn{3}{|l|}{ Location of tumor } \\
\hline Upper third & $0(0)$ & $0(0)$ \\
\hline Middle third & $0(0)$ & $0(0)$ \\
\hline \multicolumn{3}{|c|}{ Lower third or gastroesophageal } \\
\hline \multicolumn{3}{|l|}{ Neoadjuvant treatment } \\
\hline Chemoradiotherapy & $9(100)$ & $20(91)$ \\
\hline None & $0(0)$ & $2(9)$ \\
\hline Lymph nodes resected & $20(13-32)$ & $19(6-38)$ \\
\hline Radical surgery & $9(100)$ & $21(95)$ \\
\hline \multicolumn{3}{|l|}{ Comorbidities } \\
\hline Hypertension & $5(56)$ & $9(41)$ \\
\hline Heart failure & $1(11)$ & $3(14)$ \\
\hline Cardiac arrhythmia & $1(11)$ & $0(0)$ \\
\hline COPD & $1(11)$ & $2(9)$ \\
\hline Diabetes mellitus & $0(0)$ & $4(18)$ \\
\hline Chronic kidney disease & $0(0)$ & $3(14)$ \\
\hline \multicolumn{3}{|l|}{ Grading according to ECCG } \\
\hline Grade 1 & $1(11)$ & \\
\hline Grade 2 & $1(11)$ & \\
\hline Grade 3 & $7(78)$ & \\
\hline \multicolumn{3}{|c|}{ Other postoperative complications } \\
\hline Respiratory failure & $2(22)$ & $1(5)$ \\
\hline Pneumonia & $1(11)$ & $1(5)$ \\
\hline Atrial fibrillation & $1(11)$ & $2(9)$ \\
\hline Other & $3(33)$ & $3(14)$ \\
\hline Hospital stay, days & $31(18-106)$ & $8(6-17)$ \\
\hline ICU stay, days & $19(6-89)$ & $1(0-7)$ \\
\hline 30-Day mortality & $0(0)$ & $0(0)$ \\
\hline
\end{tabular}

Data are $n(\%)$ and median (range).

$\mathrm{AL}$, anastomotic leakage; COPD, chronic obstructive pulmonary disease; ECCG, Esophagectomy Complications Consensus Group; ICU, intensive care unit.

\section{Results}

\section{Patient Characteristics}

Between March 2015 and April 2016, 66 patients were eligible for inclusion. Three patients were excluded due to intraoperative findings of irresectable tumors or distant metastases. A total of 63 patients were included, undergoing esophagectomy $(n=31)$ or pancreaticoduodenectomy $(n=$ $32), 14$ of whom developed AL (24\%) postoperatively. Urine samples were collected on the first 3 postoperative days, resulting in a total of 184 samples as 5 samples were lost.

\section{Esophagectomy}

Thirty-one patients undergoing esophagectomy were included, 25 men and 6 women had a median age of 63 years (40-81). Nine patients (29\%) developed postoperative AL and median time to clinical presentation was 8 days (interquartile range 17.5). All patients developed AL within the postoperative admission, except for 1 delayed AL, which was diagnosed upon readmission after 30 days postoperatively. Grade 1 and 2 were seen in 1 patient and grade 3 was observed in 7 patients. A total of 89 urine samples were collected, 3 missing samples in the leakage group and 1 missing sample in the uncomplicated controls. Further subject characteristics are summarized in Table 1. No statistically significant differences were present between groups. Urinary VOCs were not able to distinguish AL from uncomplicated patients, AUROC 0.51 (95\% CIs 0.37-0.65). Results of the random forest analysis for both types of surgery are displayed in Table 2.

\section{Pancreaticoduodenectomy}

A total of 32 patients underwent pancreaticoduodenectomy - 23 men and 9 women - who had a median age of 66 years with a range of 45-82 years. Postoperative leakage of the pancreaticojejunostomy was observed in 6 patients (19\%), consisting of 3 level B and 3 level C AL, median time to diagnosis was 5 days (interquartile range 14.25). In 1 case, a delayed AL was diagnosed upon readmission on 30 days after surgery. Leakage of the gastrojejunostomy or hepaticojejunostomy was not observed. A single sample was not collected in the AL group, resulting in a total of 95 urine samples. Characteristics of $\mathrm{AL}$ patients and uncomplicated patients are depicted in Table 3, no statistically significant differences were found between groups. Further analysis showed that urinary VOCs were able to distinguish AL from uncomplicated patients following pancreaticoduodenectomy with a sensitivity of 0.76 (95\% CI $0.50-0.93)$ and specificity of 0.77 (95\% CI 0.66-0.86). The AUROC was 0.85 (95\% CI $0.76-$ 0.93 ) and is displayed in Figure 2. For each sample, the predicted probability of AL was determined. Figure 3 shows the difference in predictive probability broken down by surgery type. The predictive probability was 
Table 2. AUC, sensitivities, and specificities of FAIMS technique for distinguishing AL from uncomplicated controls for both types of surgery

\begin{tabular}{llll}
\hline Type of surgery & AUC (95\% CI) & Sensitivity (95\% CI) & Specificity (95\% CI) \\
\hline Pancreaticoduodenectomy & $0.85(0.76-0.93)$ & $0.76(0.50-0.93)$ & $0.77(0.66-0.86)$ \\
& $p<0.00001$ & $p<0.001$ & $p<0.001$ \\
Esophagectomy & $0.51(0.37-0.65)$ & $0.54(0.33-0.74)$ & $0.55(0.42-0.67)$ \\
& $p=0.88$ & $p=0.48$ & $p=0.48$ \\
\hline
\end{tabular}

$p$ values are calculated by means of the Mann-Whitney $U$ test for AUC, and with the Fisher's exact test for sensitivity and specificity.

AUC, area under the curve; FAIMS, field asymmetric ion mobility spectrometry; AL, anastomotic leakage.

broken down by postoperative day following pancreaticoduodenectomy in Figure 4 and revealed that there does not appear to be a tendency for individual postoperative day samples to be more associated with AL. Samples collected on postoperative day 3 appeared to be more consistently scored as low risk for AL compared to other postoperative days.

\section{Discussion}

In the present pilot study, urinary VOCs of patients undergoing pancreaticoduodenectomy could predict AL correctly at an early stage. Urine samples were collected during the first 3 postoperative days, while the median time to clinical presentation for pancreatic AL was about 5 days. Preclinical detection could enable early intervention (percutaneous drainage or laparotomy), possibly leading to better outcome and timely start of adjuvant treatment. The results suggest that non-invasive urine analysis by means of FAIMS can be of value in the early, preclinical detection of AL following pancreatic surgery. However, this was not observed in patients undergoing esophagectomy.

This study is the first to our knowledge to use VOC analysis to diagnose postoperative AL. Leakage is frequently accompanied by, or the result of, anastomotic ischemia. This creates localized biochemical and immunological changes. These changes consist of decreasing glucose and pyruvate levels and increasing lactate and glycerol levels. Mediastinal microdialysis has been shown to be a promising method to detect these biochemical changes before the development of significant clinical symptoms [19]. Furthermore, during the inflammatory response, other endogenous products are produced and present themselves as VOCs. These VOCs defuse into the blood, are renally excreted, and can be detected in urine samples [20, 21]. In current practice, inflammation markers as serum CRP are frequently used to detect AL. Research has shown that postoperative CRP levels rise in response to the initial surgery and show a physiological peak approximately 48-72 h postoperatively. This hampers the use of CRP in this phase.

Urinary VOCs of patients following esophagectomy could not predict AL. Median time to clinical presentation of AL and the leaked content might have been important factors why the technique appears to be accurate following pancreaticoduodenectomy but not esophagectomy. The median time to clinical presentation of esophageal AL was 8 days, compared to 5 days for pancreatic AL. Therefore, the preclinical biochemical and immunological changes related to AL were possibly not detectable during the first 3 postoperative days following esophagectomy. Furthermore, pancreatic AL is associated with leakage of pancreatic enzymes. Increased levels of pancreatic enzymes in drain fluid have shown to be predictive for pancreatic AL (6). These enzymes might have contributed to the accuracy of the technique following pancreatic surgery.

In general, 3 analytical methods are used to detect gas phase VOCs: gas chromatography combined with mass spectrometry (GC-MS), electronic nose devices, and IMS. Urinary VOC analysis was performed using FAIMS, which has a number of advantages over other techniques. GC-MS is considered the primary method to detect individual VOCs. However, analysis by means of GC-MS is costly and time consuming, making it less feasible for the clinical setting. Compared with traditional electronic noses, using an array of nanosensors and pattern recognition, FAIMS has the advantage of a higher sensitivity (i.e., parts per billion to parts per trillion) and an absence of sensor drift over time [22], due to the measurement of physical, not chemical, properties of the volatile molecules. 
Table 3. Summary of demographic and clinical characteristics of patients undergoing pancreatic surgery

\begin{tabular}{|c|c|c|}
\hline Characteristics & $\begin{array}{l}\mathrm{AL} \\
(n=6)\end{array}$ & $\begin{array}{l}\text { Controls } \\
(n=26)\end{array}$ \\
\hline \multicolumn{3}{|l|}{ Gender } \\
\hline Male & $5(83)$ & $18(69)$ \\
\hline Female & $1(17)$ & $8(31)$ \\
\hline Age, years & $69(58-78)$ & $68(45-82)$ \\
\hline Missing urine samples & $1(6)$ & $0(0)$ \\
\hline \multicolumn{3}{|l|}{ Type of resection } \\
\hline Pancreaticoduodenectomy & $3(50)$ & $20(77)$ \\
\hline PPPD & $3(50)$ & $6(23)$ \\
\hline \multicolumn{3}{|l|}{ Primary tumor } \\
\hline Pancreas & $3(50)$ & $14(54)$ \\
\hline Ampulla of Vater & $1(17)$ & $4(15)$ \\
\hline Duodenum & $1(17)$ & $2(8)$ \\
\hline Biliary & $0(0)$ & $5(19)$ \\
\hline No malignancy & $1(17)$ & $1(4)$ \\
\hline Lymph nodes resected & $7(4-14)$ & $10(3-26)$ \\
\hline Radical surgery & $4(67)$ & $14(54)$ \\
\hline \multicolumn{3}{|l|}{ Comorbidities } \\
\hline Hypertension & $3(50)$ & $15(58)$ \\
\hline Heart failure & $1(17)$ & $2(8)$ \\
\hline Cardiac arrhythmia & $0(0)$ & $4(15)$ \\
\hline COPD & $0(0)$ & $4(15)$ \\
\hline Diabetes mellitus & $1(17)$ & $6(23)$ \\
\hline Chronic kidney disease & $0(0)$ & $0(0)$ \\
\hline \multicolumn{3}{|l|}{ Grading according to ISGPF } \\
\hline Grade A & $0(0)$ & \\
\hline Grade B & $1(17)$ & \\
\hline Grade C & $5(83)$ & \\
\hline \multicolumn{3}{|c|}{ Other postoperative complications } \\
\hline Gastroparesis & $2(33)$ & $3(12)$ \\
\hline Chyle leakage & $1(17)$ & $4(15)$ \\
\hline Postoperative hemorrhage & $1(17)$ & $2(8)$ \\
\hline Pneumonia & $1(17)$ & $1(4)$ \\
\hline Other & $2(33)$ & $7(27)$ \\
\hline Hospital stay, days & $48(10-95)$ & $11(4-38)$ \\
\hline ICU stay, days & $4(1-88)$ & $0(0-11)$ \\
\hline 30-Day mortality & $0(0)$ & $0(0)$ \\
\hline
\end{tabular}

Data are $n(\%)$ and median (range).

$\mathrm{AL}$, anastomotic leakage; PPPD, pylorus-preserving pancreaticoduodenectomy; COPD, chronic obstructive pulmonary disease; ISGPF, International Study Group on Pancreatic Fistula; ICU, intensive care unit.

In previous gastrointestinal research, feces and exhaled breath have frequently been used for VOC analysis $[14,23]$. Metabolites of infectious processes in the gastrointestinal tract, such as AL, are discharged in feces. Therefore, it could be hypothesized that the analysis of fecal VOCs is more appropriate to detect $\mathrm{AL}$ compared to urine. However, collection of feces was considered less feasible, due to possible temporary im-

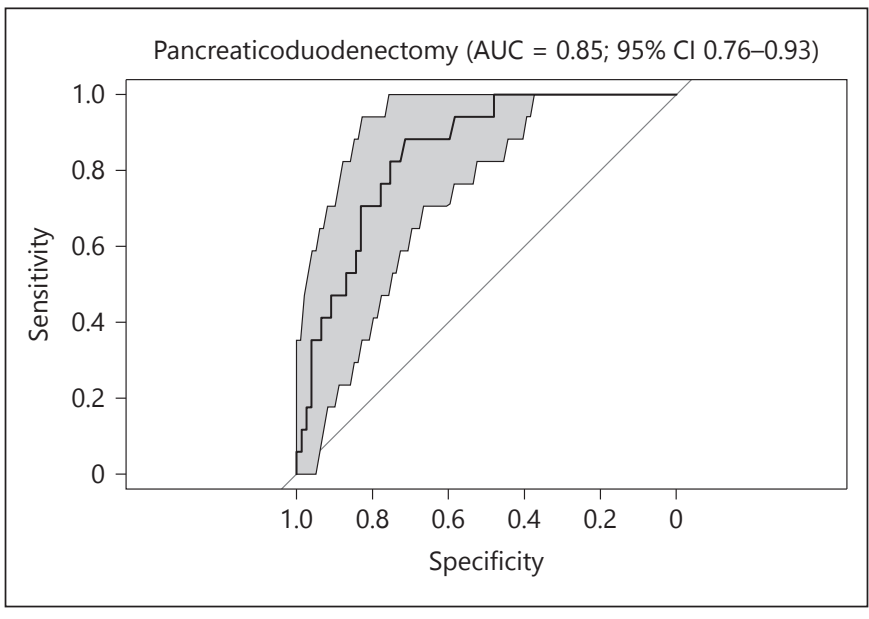

Fig. 2. Receiver operator curve plots for AL vs. uncomplicated controls following pancreaticoduodenectomy. AL, anastomotic leakage; AUC, area under the curve.

pairment of bowel motility in patients undergoing major gastrointestinal surgery during the early postoperative phase. Furthermore, urine was preferred over exhaled breath, due to the costs and logistical challenges of collection, storage, and analysis of exhaled breath.

The greatest limitation of this explorative study is the small sample size. However, despite low numbers and a substantial heterogeneity within the study population, VOC analysis by FAIMS allowed for discrimination between AL and uncomplicated patients following pancreaticoduodenectomy. Due to the small cohort, it was necessary to pool the data across days for each type of surgery when performing the analysis. Since each patient in the study contributed multiple urine samples, and all samples from a given patient have the same AL status, this introduces a potential bias to the results: if the training data contains samples 1 and 2 from patient $A$, and the test data contains sample 3 from patient $A$, then there is a danger that instead of detecting a VOC signature for AL, a VOC signature for each patient is detected and that signature is used to assign AL status to the samples in the test data. However, if this effect were causing erroneous positive results a positive result would have been seen for both the esophageal and pancreatic cohorts. Since a positive result was only observed for the pancreatic cohort, it can be inferred that the bias introduced by this method is not sufficient to invalidate the positive result observed. Furthermore, the small sample size prevented us from assessing the potential influence of comorbidities, other postoperative complications, 
Fig. 3. Analysis of the urinary VOCs resulted in a predicted probability of AL for each sample. This figure shows the predicted probability of AL broken down by surgery type and true AL status. AL, anastomotic leakage.

Fig. 4. Predicted probability of AL following pancreaticoduodenectomy broken down by postoperative day and true AL status. AL, anastomotic leakage.
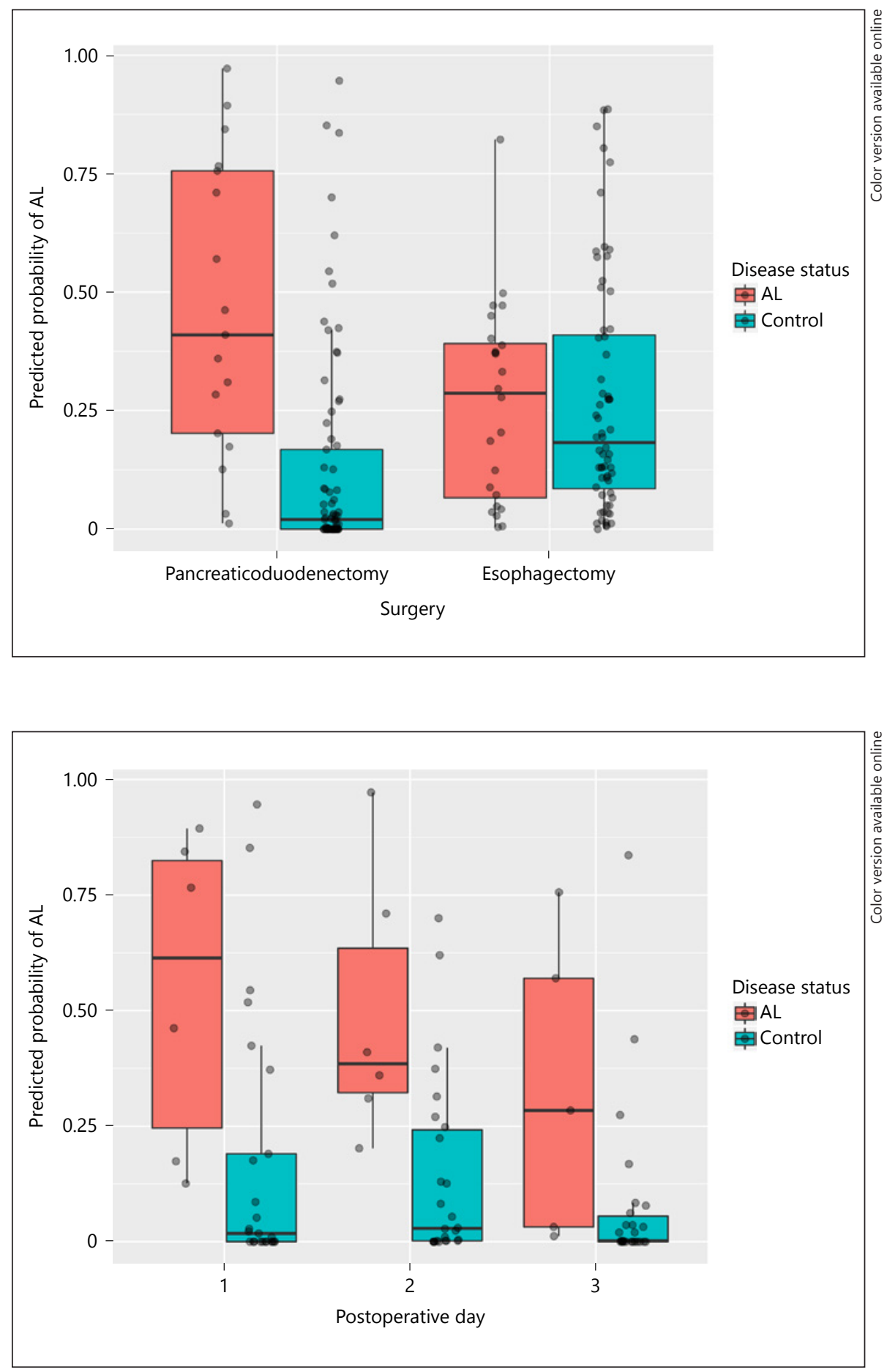

varying disease stages, and types of cancer requiring neo-adjuvant chemotherapy or chemoradiotherapy. Further research is justified to address these limitations and should focus on improving the accuracy of FAIMS and use other analytical methods, such as GC-MS to identify individual VOC.

Non-Invasive Detection of AL
In conclusion, in our study population, urinary VOC analysis allowed for discrimination between $\mathrm{AL}$ and controls following pancreaticoduodenectomy on all 3 postoperative days combined. VOC analysis could not distinguish AL from controls following esophagectomy. 


\section{References}

-1 Turrentine FE, Denlinger CE, Simpson VB, Garwood RA, Guerlain S, Agrawal A, et al: Morbidity, mortality, cost, and survival estimates of gastrointestinal anastomotic leaks. J Am Coll Surg 2015;220:195-206.

2 Dutch Institute for Clinical Auditing. Annual Reports. http://www.dica.nl.

-3 Straatman J, Cuesta MA, Gisbertz SS, Van der Peet DL: Value of a step-up diagnosis plan: CRP and CT-scan to diagnose and manage postoperative complications after major abdominal surgery. Rev Esp Enferm Dig 2014; 106:515-521.

-4 Straatman J, Harmsen AM, Cuesta MA, Berkhof J, Jansma EP, van der Peet DL: Predictive value of $\mathrm{C}$-reactive protein for major complications after major abdominal surgery: a systematic review and pooled-analysis. PLoS One 2015;10:e132995.

5 Perry Y, Towe CW, Kwong J, Ho VP, Linden PA: Serial drain amylase can accurately detect anastomotic leak after esophagectomy and may facilitate early discharge. Ann Thorac Surg 2015;100:2041-2047.

6 Reid-Lombardo KM, Farnell MB, Crippa S, Barnett M, Maupin G, Bassi C, et al: Pancreatic anastomotic leakage after pancreaticoduodenectomy in 1,507 patients: a report from the pancreatic anastomotic leak study group. J Gastrointest Surg 2007;11:1451-1458.

7 Nagakawa Y, Matsudo T, Hijikata Y, Kikuchi S, Bunso K, Suzuki Y, et al: Bacterial contamination in ascitic fluid is associated with the development of clinically relevant pancreatic fistula after pancreatoduodenectomy. Pancreas 2013;42:701-706.

8 de Groot EF, de Meij TG, Berkhout DJ, van der Schee MP, de Boer NK: Flatography: detection of gastrointestinal diseases by faecal gas analysis. World J Gastrointest Pharmacol Ther 2015;6:111-113.

-9 Turner AP, Magan N: Electronic noses and disease diagnostics. Nat Rev Microbiol 2004; 2:161-166.

10 Sonoda H, Kohnoe S, Yamazato T, Satoh Y, Morizono G, Shikata K, et al: Colorectal cancer screening with odour material by canine scent detection. Gut 2011;60:814-819.

11 Horvath I, Lazar Z, Gyulai N, Kollai M, Losonczy G: Exhaled biomarkers in lung cancer. Eur Respir J 2009;34:261-275.

-12 Buszewski B, Kesy M, Ligor T, Amann A: Human exhaled air analytics: biomarkers of diseases. Biomed Chromatogr 2007;21:553-566.

13 Arasaradnam RP, Westenbrink E, McFarlane MJ, Harbord R, Chambers S, O'Connell N, et al: Differentiating coeliac disease from irritable bowel syndrome by urinary volatile organic compound analysis - a pilot study. PLoS One 2014;9:e107312.

14 Arasaradnam RP, McFarlane MJ, Ryan-Fisher C, Westenbrink E, Hodges P, Thomas MG et al: Detection of colorectal cancer (CRC) by urinary volatile organic compound analysis. PLoS One 2014;9:e108750.

15 Low DE, Alderson D, Cecconello I, Chang AC, Darling GE, D'Journo XB, et al: International consensus on standardization of data collection for complications associated with esophagectomy: esophagectomy complications consensus group (ECCG). Ann Surg 2015;262:286-294.

16 Bassi C, Marchegiani G, Dervenis C, Sarr M, Abu Hilal M, Adham M, et al: The 2016 update of the international study group (ISGPS) definition and grading of postoperative pancreatic fistula: 11 Years After. Surgery 2017; 161:584-591.
17 Covington JA, van der Schee MP, Edge AS, Boyle B, Savage RS, Arasaradnam RP: The application of FAIMS gas analysis in medical diagnostics. Analyst 2015;140:6775-6781.

$\longrightarrow 18$ Esfahani S, Sagar NM, Kyrou I, Mozdiak E, O'Connell N, Nwokolo C, et al: Variation in gas and volatile compound emissions from human urine as it ages, measured by an electronic nose. Biosensors (Basel) 2016;6:pii:E4.

19 Ellebaek M, Qvist N, Fristrup C, Mortensen MB: Mediastinal microdialysis in the diagnosis of early anastomotic leakage after resection for cancer of the esophagus and gastroesophageal junction. Am J Surg 2014;208: 397-405.

20 Arasaradnam RP, Ouaret N, Thomas MG, Quraishi N, Heatherington E, Nwokolo CU, et al: A novel tool for noninvasive diagnosis and tracking of patients with inflammatory bowel disease. Inflamm Bowel Dis 2013;19: 999-1003.

21 Sethi S, Nanda R, Chakraborty T: Clinical application of volatile organic compound analysis for detecting infectious diseases. Clin Microbiol Rev 2013;26:462-475.

22 Covington JA, Wedlake L, Andreyev J, Ouaret N, Thomas MG, Nwokolo CU, et al: The detection of patients at risk of gastrointestinal toxicity during pelvic radiotherapy by electronic nose and FAIMS: a pilot study. Sensors (Basel) 2012;12:13002-13018.

-23 de Meij TG, Larbi IB, van der Schee MP, Lentferink YE, Paff T, Terhaar Sive Droste JS, et al: Electronic nose can discriminate colorectal carcinoma and advanced adenomas by fecal volatile biomarker analysis: proof of principle study. Int J Cancer 2014;134:11321138. 